\title{
Validación transcultural de la Escala de Inteligencia Emocional de Schutte*
}

\author{
Cross-cultural validation of the \\ Schutte Emotional Intelligence Scale
}

\begin{abstract}
Alicia Omar*
Solana Salessi

Florencia Urteaga

Juan Diego Vaamonde

Consejo Nacional de Investigaciones Científicas y Técnicas (CONICET) Universidad Nacional de Rosario Argentina

Recibido: 8 de diciembre de 2012 Revisado: 10 de febrero de 2013 Aceptado: 18 de abril de 2013
\end{abstract}

Resumen

El objetivo del estudio fue adaptar la Escala de Inteligencia Emocional de Schutte para su empleo con adolescentes y jóvenes. Se efectuó la equivalencia conceptual, semántica y operacional de la versión prototípica del instrumento. La versión definitiva fue administrada a una muestra de 1.890 jóvenes y adolescentes (730 mexicanos, 664 argentinos y 497 brasileros). El análisis de la validez factorial mostró una estructura de dos factores que explicaron el $63.9 \%$ de la varianza total. La validez convergente quedó demostrada a partir del análisis correlacional efectuado. La confiabilidad del instrumento alcanzó niveles satisfactorios. Se efectúan sugerencias para futuras investigaciones.

Palabras clave: escala de inteligencia emocional, validación estadística, adolescentes, jóvenes, latinoamericanos. 


\section{Abstract}

The aim of this study was to adapt the Schutte Emotional Intelligence Scale with Latinamerican adolescents and youth adults. The conceptual, semantic, and operational equivalence of the prototype version was analyzed. The final version was administered to a sample of 1.890 students (730 Mexicans, 664 Argentineans, and 497 Brazilians). The analysis of factorial validity showed two orthogonal factors which explained $63.9 \%$ of the total variance. Convergent validity was demonstrated through the correlation analysis performed. The reliability of the adapted instrument reached satisfactory levels. In light of the results, we offer some suggestions for future research in the area.

Keywords: Emotional intelligence scale, statistical validation, adolescents, youth, Latin Americans.

\section{Introducción}

El empleo del término inteligencia emocional (IE) puede rastrearse en la bibliografía científica desde antes de 1990, aunque es a partir de esa fecha cuando irrumpe en el ámbito académico (Salovey y Mayer, 1990) y alcanza notoriedad tras la publicación del libro de Goleman (1995). El crecimiento exponencial del interés por el constructo no se ha visto acompañado por el consenso acerca de cómo debe ser definido y operacionalizado. Esta situación ha propiciado la emergencia de dos modelos explicativos, identificados como modelo mixto y modelo de habilidad cognitiva, respectivamente (Mayer, Roberts y Barsade, 2008).

El modelo mixto (Bar-On, 2005), al que adhiere Goleman (1995), concibe a la IE como una combinación de rasgos de personalidad y motivación. Los críticos de esta perspectiva (Roberts, MacCann, Matthews y Zeidner, 2010; Van Rooy, Whitman y Viswesvaran, 2010), argumentan que las escalas desarrolladas para medir la IE se solapan con algunas medidas de personalidad, lo que contribuye a disminuir su validez discriminante. El modelo de habilidad cognitiva (Mayer y Salovey, 1993; Mayer, Salovey y Caruso, 2002), considera a la IE como un conjunto de habilidades para percibir, valorar y expresar emociones adaptativamente; entender las emociones y cogniciones emocionales, y regular los afectos para promover el crecimiento intelectual (Fiori y Antonakis, 2011).
Frente a esta dicotomía, Petrides (2000) y Furham (2003) sostienen que más que diferenciar entre dos modelos teóricos, se debe discriminar en función de la operacionalización del constructo. Por lo que proponen distinguir entre "IE rasgo" e "IE habilidad". La IE habilidad ocupa el dominio cognitivo y es medida a través de tests de habilidad conformados por ítems con respuestas correctas o incorrectas. La IE rasgo incorpora un amplio espectro de características de personalidad y otros rasgos, y es medida a través de instrumentos autodescriptivos.

Los cultores de la IE habilidad han desarrollado algunos instrumentos inspirados en las pruebas objetivas de rendimiento, similares a los tradicionales tests de inteligencia. Los más difundidos son la Escala Multifactorial de Inteligencia Emocional (Mayer, Caruso y Salovey, 1999) y el Inventario Mayer-Salovey-Caruso (MSCEIT; Mayer, Salovey y Caruso, 2002), que constituye una versión superadora del primero. El MSCEIT mide cuatro dimensiones de la IE (percepción, uso, entendimiento y regulación emocional) a través de 141 ítems, posee adecuada validez discriminante y convergente (Mayer, Salovey y Caruso, 2012) y, en la actualidad, es el más utilizado para medir la IE habilidad. En la práctica, estos instrumentos se han enfrentado con algunos inconvenientes, tales como dificultades para justificar como "correctas" las respuestas vinculadas a cuestiones emocionales. Los partidarios de la IE rasgo, por su parte, han elaborado diversas escalas autodescriptivas, en- 
tre las que se destacan el Inventario de Cociente Emocional (EQ-i), desarrollado por Bar-On (1997), cuyos 133 ítems miden las cinco competencias emocionales y las quince habilidades postuladas en su modelo teórico; y la Escala de Inteligencia Emocional (SSREI, Schutte Self Report Emotional Intelligence Scale), desarrollada por Schutte y sus colegas (1998), de la que se ocupa el presente trabajo. Esta escala está integrada por 33 ítems, diseñados para medir las dimensiones de evaluación, regulación y uso de las emociones desde la perspectiva de la IE rasgo (Salovey y Mayer, 1990). Inicialmente, Schutte y sus colaboradores elaboraron 62 ítems, los que aplicaron a una muestra de 346 estudiantes universitarios norteamericanos. A partir de un análisis de componentes principales, seguido de una rotación varimax, extrajeron cuatro factores, de los que solo retuvieron el primero porque era el más parsimonioso. Por lo que la escala de Schutte quedó definida como una medida unidimensional para evaluar el factor general de IE, con un alfa de .87 y una confiabilidad test-retest de .78. La validez convergente de la escala fue establecida a través de correlaciones con medidas de habilidad para regular las emociones, depresión e impulsividad.

Desde su aparición, numerosos estudios han analizado las propiedades psicométricas de la SSREI, mostrando mayor consenso con respecto a la validez convergente que a la validez estructural del intrumento. Para el establecimiento de la validez convergente, se han tomado en cuenta las especulaciones teóricas que indican que la IE facilita el afrontamiento del estrés (Davis y Humphrey, 2012), la resiliencia (Liu, Wang y Lü, 2013), la adaptación psicosocial y el bienestar (Bhullar, Schutte y Malouff, 2013), entre otros efectos benéficos.

En lo que hace a la validez factorial se han llevado a cabo, al menos, una decena de estudios, con resultados dispares. Petrides y Furnham (2000) fueron los primeros en examinar la estructura de la escala de SSREI. Comunicaron una solución tetrafactorial, aunque concluyeron recomendando mayor investigación de la estructura factorial antes de usar el instrumento. Ciarrochi, Chan y Caputi (2000) obtuvieron resultados similares a los de Petrides y Furnham, con pequeñas diferencias vin- culadas con la redacción de dos ítems. Brackett y Mayer (2003) identificaron un solo factor interpretable (percepción o evaluación de la emoción), en sintonía con el modelo unidimensional definido por Schutte et al. (1998). Austin, Saklofske, Huang y McKenney (2004) comunicaron una solución trifactorial, luego de aumentar el número de ítems negativamente redactados, ya que una de las mayores críticas a la SSREI es su escaso número (solo tres) de ítems reversos.

A través de un análisis factorial exploratorio con rotación varimax, Chan (2003) encontró que la solución de cuatro factores era la más interpretable, luego de descartar 5 ítems porque no presentaban cargas adecuadas sobre ningún factor. Hakanen (2004) también obtuvo una solución tetrafactorial, aunque sus factores no quedaron definidos por los mismos ítems informados por los estudios previos. Gignac, Palmer, Manocha y Stough (2005) señalaron que el estudio de Hakanen (2004), presentaba las mismas limitaciones de otros estudios factoriales de la SSREI, dado que empleaba rotaciones ortogonales cuando debería haberse usado rotaciones oblicuas, ya que los factores deben estar conceptualmente relacionados. Antes de analizar la estructura de la SSREI, Gignac et al. (2005) categorizaron cualitativamente los 33 ítems de la escala en las dimensiones conceptuales definidas por Salovey y Mayer (1990). Sus análisis se basaron en 28 ítems, ya que no lograron categorizar 5 ítems en ninguna de las dimensiones establecidas. Luego de varias modificaciones y eliminaciones de nuevos ítems, Gignac et al. (2005) presentaron un modelo anidado de 6 factores con 21 ítems. Más recientemente, Saklofske, Austin, Mastoras, Beaton y Osborne (2012) efectuaron análisis factoriales exploratorios con rotación oblicua. Luego de varias modificaciones a los ítems propusieron un modelo tetrafactorial, aunque concluyeron señalando la necesidad de mayores estudios de la estructura factorial de la SSREI. Similares resultados fueron comunicados por García-Coll, GrauperaSanz, Ruiz-Pérez y Palomo-Nieto (2013), después de eliminar los tres ítems redactados negativamente.

Como se observa, la mayoría de las verificaciones realizadas no coincide con respecto a la estructura 
factorial de la SSREI y, en términos generales, los autores recomiendan más investigaciones en este sentido. En los estudios factoriales realizados se han empleado diferentes poblaciones (canadienses, ingleses, chinos, españoles), aunque hasta la fecha la escala no ha sido validada para su empleo con jóvenes y adolescentes latinoamericanos, por lo que se trata de una tarea pendiente. Sobre todo porque la verificación de la invariancia factorial de una medida en muestras diferentes a las ya estudiadas es fundamental para la ampliación de su validez externa, así como para establecer su utilidad para diferentes propósitos (Asociación Americana de Psicología, 1999). De allí que el propósito del presente trabajo fue adaptar y validar la escala de IE desarrollada por Schutte y colegas (1998) para su empleo con adolescentes y jóvenes latinoamericanos.

\section{Método}

De las diferentes formas de adaptación disponibles, se optó por el método analítico secuencial propuesto por Herdman, Fox-Rushby y Badía (1998). Este método consta de un conjunto de pasos (equivalencia conceptual, semántica, operacional y de medición), los que se describen a continuación.

La equivalencia conceptual se refiere a la equivalencia de cada concepto en la cultura donde fue desarrollado el instrumento con la cultura objetivo (en la que se aplicará el instrumento). En el presente estudio se efectuó una exhaustiva revisión bibliográfica sobre el tema IE tanto en la cultura original del instrumento (Estados Unidos de Norteamérica) como en la lusohispánica. De particular utilidad en esta etapa fue la evaluación crítica del constructo realizada por Ciarrochi y colegas (2000), la que indica que la IE se asocia en la satisfacción con la vida y con la calidad de las relaciones sociales en todas las culturas. Simultáneamente, los ítems fueron sometidos a una intensa revisión crítica con el propósito de determinar su adecuación a la edad del grupo objetivo (jóvenes y adolescentes). La evaluación y juicio de especialistas indicaron que el espectro explorado por el instrumento cubría los dominios de la
IE postulados por Schutte, entre los jóvenes y los adolescentes latinoamericanos.

La equivalencia semántica consiste en la traducción del instrumento conservando el significado entre idiomas diferentes. En este caso, el análisis de la equivalencia semántica fue realizado en cuatro etapas: (a) el instrumento original fue traducido del inglés al español (argentino y mexicano) y al portugués (brasilero); (b) expertos en inglés retradujeron ambas versiones al inglés; (c) traductores de inglés compararon a ciegas las dos formas del instrumento a efectos de identificar la concordancia entre el ítem original y el traducido; (d) tomando en cuenta las apreciaciones de los especialistas los autores del presente trabajo discutieron y decidieron la inclusión de los ítems que integrarían las versiones españolas (argentino-mexicana) y portuguesa (brasilera) del instrumento. Tales versiones prototípicas fueron administradas a una muestra por disponibilidad de la población objetivo integrada por 420 estudiantes (Argentina $=180$, Brasil $=101$ y México $=172)$, con una edad promedio de 18.25 años (desvío típico= 3.62). La finalidad de este estudio piloto fue explorar la equivalencia operacional (mantenimiento de las características operativas del universo original mediante el empleo de ciertas normas antes y durante la aplicación de los instrumentos), básicamente en lo referente a tiempo para responder la escala, claridad de las instrucciones para efectuar la tarea y adecuación semántica y sintáctica de los ítems. Este estudio piloto demostró que los ítems eran bien comprendidos, que las instrucciones estaban claramente redactadas y que la escala elegida para responder el inventario (Likert de 5 puntos) no generaba dificultades.

La equivalencia de medición tiene como propósito analizar las propiedades psicométricas de un instrumento a través del cálculo de medidas de confiabilidad y validez. En este caso, para establecer la validez estructural de la escala, se realizó un análisis factorial exploratorio, seguido de un análisis confirmatorio. La validez convergente se determinó a través de la correlación con otros constructos que, a partir de la revisión de la literatura especializada, surgían como relevantes con respecto a la IE. La confiabilidad se calculó me- 
diante el cómputo de coeficientes de consistencia interna (Alpha de Cronbach) y de estabilidad test-retest. Para el estudio de la equivalencia de medición se empleó la muestra y los instrumentos que se describen a continuación:

\section{Participantes}

Las versiones españolas y portuguesas de la SSREI puestas a punto durante la prueba piloto, fueron aplicadas a una muestra de 1.890 estudiantes (Brasil $=497$, Argentina $=663$ y México $=730$ ), de los cuales 1.108 eran mujeres y 783 varones, dentro de un rango de edad de 15 a 26 años. La media de edad de los paticipantes fue de 19.90 con un desvío típico de 2.49 , lo que está indicando que las edades centrales (que representan algo más de la mitad de la muestra) oscilaron entre los 17 y los 22 años, quedando los grupos de edades extremas, reducidos a porcentajes menores al $20 \%$. Se trató de una muestra por disponibilidad de los alumnos matriculados en escuelas y universidades públicas y privadas de sus respectivos países. La muestra brasilera estuvo integrada por estudiantes de la región metropolitana del estado de Río de Janeiro (60\% de la ciudad de Río de Janeiro; 15 $\%$ de Nova Iguaçu; $15 \%$ de Belford Roxo y $10 \%$ de Seropédica). La muestra argentina estuvo integrada por estudiantes de la zona centro del país (50 $\%$ de la ciudad de Rosario, $20 \%$ provenientes de localidades próximas a Rosario y el 30 \% restante de la ciudad de Paraná). La muestra mexicana se integró con un $70 \%$ de alumnos radicados en la zona central del país (50\% en el Distrito Federal y un $20 \%$ en Tlanepantla y Naucalpan, municipios localizados en la zona metropolitana del Distrito Federal; un $15 \%$ radicados en Mérida, y el restante $15 \%$ proveniente de Monterrey). En todos los casos los participantes fueron contactados en sus lugares de clase y luego de hacerles conocer los objetivos de la investigación fueron invitados a participar en la misma. Solo participaron aquellos que aceptaron hacerlo voluntariamente después de firmar un protocolo de consentimiento informado. En todos los casos se les garantizó el anonimato y la confidencialidad de la información suministrada.

\section{Intrumentos}

Los participantes respondieron un cuadernillo preparado en su idioma oficial (español para argentinos y mexicanos, y portugués para brasileros) compuesto por la SSREI (33 ítems con formato Likert de 5 puntos, variando desde "nunca" a "siempre") y una selección de escalas y subescalas, a saber:

Bienestar subjetivo. Se emplearon tres subescalas de la adaptación transcultural (Omar, Paris, Aguiar, Almeida y Pino Peña, 2009) del Inventario de Bienestar Subjetivo de Nacpal y Shell (1992). Las dimensiones seleccionadas fueron las de Bienestar vinculado con expectativas y logros (ejemplo: ¿estás satisfecho con las cosas que has venido haciendo en los últimos años?; 6 ítems, $\alpha=.74$ ), Confianza para afrontar las dificultades (ejemplo: ¿sientes que puedes manejar situaciones inesperadas?; 3 ítems, $a=.70)$ y Relaciones con el grupo primario (ejemplo: ¿estás conforme con la relación que tienes con tus padres?; 3 ítems, $\alpha=.80$ ). Todas las escalas están integradas por ítems con formato Likert de 5 puntos, variando desde "nunca" a siempre".

Afrontamiento del estrés. Se midió a través de las subescalas de Estrategias enfocadas en el problema (ejemplo: me dedico a resolver el problema poniendo en juego todas mis capacidades; $\mathrm{a}=.81$ ) y Estrategias adaptativas (ejemplo: hablo con otros sobre mi problema para que me ayuden a resolverlo; $a=.78$ ), de la Escala de Afrontamiento del Estrés para Adolescentes de Frydenberg y Lewis (1997). Ambas subescalas están integradas por 4 ítems cada una, con formato Likert de 5 puntos, variando desde "nunca" a siempre". En el presente estudio, y con el propósito de examinar la validez de constructo de los ítems aplicados, se realizó un análisis factorial confirmatorio. Los resultados mostraron que un modelo bifactorial $\left(\chi_{(268)}^{2}=1375.72 ; p=.000 ; C F I=.97 ; T L I=.91 ; R M-\right.$ $\mathrm{SEA}=.05$ ) era el que presentaba el mejor ajuste.

Resiliencia. Se empleó la adaptación transcultural (Almeida, Aguiar, Monteiro y Omar, 2007) de la Escala de Resiliencia (Wagnild y Young, 1993), integrada por 12 ítems con formato Likert de 5 puntos 
( $1=$ totalmente en desacuerdo; $5=$ totalmente de acuerdo), distribuídos sobre tres subescalas: Realización Personal (ejemplo: me siento orgulloso de haber conseguido cosas en la vida; $\alpha=.87$ ); Autodeterminación (ejemplo: puedo superar momentos difíciles porque ya he pasado por dificultades; $\mathrm{a}=.82$ ) y Autoconfianza (ejemplo: soy capaz de valerme por mí mismo; $\alpha=.91$ ).

Sentido del humor. Se empleó la adaptación transcultural (Almeida et al. 2007) de las subescalas de Generación y usos sociales del humor (ejemplo: la gente me considera capaz de decir cosas divertidas; $\alpha=.84)$ y Usos del humor como mecanismo de coping (ejemplo: el humor me ayuda a manejar las situaciones difíciles; $\alpha=.97$ ), de la Escala Multidimensional del Sentido del Humor de Thorson y Powell (1993). Cada subescala está integrada por 5 ítems con formato Likert de 5 puntos, variando desde "muy en desacuerdo" a "muy de acuerdo".

El cuadernillo se completó con un conjunto de datos sociodemográficos (edad, género, nivel educacional y tipo de establecimiento educativo) de cada participante.

\section{Análisis de datos}

Se calcularon análisis de la varianza para comprobar los supuestos de invarianza métrica entre las muestras argentinas, brasileras y mexicanas. Se efectuaron análisis factoriales exploratorios (AFE) para identificar la estructura factorial de la escala, y análisis factoriales confirmatorios (AFC) para analizar la adecuación de los datos con el mode- lo identificado. En la ejecución de los AFC (Hair, Black, Babin y Anderson, 2009) se usó el método de estimación de la máxima verosimilitud, que proporciona medidas de bondad de ajuste entre el modelo propuesto y los datos empíricos recogidos. Los índices de ajustes analizados fueron los siguientes: Chi cuadrado relativo (CMIN/DF), índice de bondad de ajuste (GFI), índice comparativo de Bentler (CFI), índice de Tucker-Lewis (TLI), y error de aproximación de la raíz cuadrada media (RMSEA). La confiabilidad de los factores identificados se determinó mediante los métodos de test-retest y de consistencia interna (Alpha de Cronbach).

\section{Resultados}

La equivalencia semántica de las versiones españolas (argentino-mexicana) y portuguesa (brasilera) de la SSREl fue determinada en función de dos categorías de análisis. Por un lado, el significado referencial (concordancia en términos de traducción literal entre el ítem original y el traducido), fue evaluado sobre una escala visual analógica en la que la equivalencia entre pares fue juzgada de 0 a $100 \%$. En este sentido, se logró una equivalencia cercana al $100 \%$ ya que ninguno de los ítems generó algún nivel de desacuerdo entre los traductores. Por otro lado, el significado general (concordancia más amplia, en términos de articulación de ideas entre el ítem original y su retraducción), fue evaluado en función de cuatro niveles de equivalencia: inalterado, poco alterado, muy alterado y completamente alterado (tabla 1).

Tabla 1.

Niveles de equivalencia semántica entre el ítem original y su retraducción.

\begin{tabular}{lcccc}
\hline \multirow{2}{*}{ Nivel de equivalencia } & \multicolumn{2}{c}{$\begin{array}{c}\text { Traducción-retraducción } \\
\text { (inglés-español-inglés) }\end{array}$} & \multicolumn{2}{c}{$\begin{array}{c}\text { Traducción-retraducción } \\
\text { (inglés-portugués-inglés) }\end{array}$} \\
\cline { 2 - 5 } Inalterado & Traductor 1 & Traductor 2 & Traductor 1 & Traductor 2 \\
\cline { 2 - 5 } Poco alterado & $27 / 33=81.82 \%$ & $28 / 33=84.85 \%$ & $30 / 33=90.91 \%$ & $28 / 33=84.85 \%$ \\
Muy alterado & $4 / 33=12.12 \%$ & $2 / 33=6.06 \%$ & $3 / 33=9.09 \%$ & $2 / 33=6.06 \%$ \\
Completamente alterado & $2 / 33=6.06 \%$ & $3 / 33=9.09 \%$ & $0 / 33=0.00 \%$ & $2 / 33=6.06 \%$ \\
\hline
\end{tabular}


Antes de comenzar el análisis de la equivalencia de medición, se exploró la invarianza de la medida entre las tres muestras nacionales a través del cálculo de un análisis de la varianza (ANOVA). Los resultados obtenidos $\left(F_{1888(2)}=.67\right.$; n.s. $)$ indicaron que no existían diferencias significativas, por lo que se consideraron a los tres subgrupos como un grupo único. Seguidamente, y sobre la muestra total integrada por 1890 jóvenes y adolescentes, se procedió a la determinación de la validez factorial de la SSREI mediante el cálculo de análisis de componentes principales con rotación oblicua. La matriz de datos fue considerada factorizable, desde el momento que el test de esfericidad de Bartlett fue significativo (Chi cuadrado aproximado= 7541.20; $g l=276 ; p<.000$ ); el test de adecuación de la muestra de Kaiser-Meyer-Olkin arrojó un valor de .92, y el cuadrado del coeficiente de correlación múltiple (MAS) fue de .94. El primer análisis ejecutado produjo una solución tetrafactorial, en la que el tercer factor solo nucleó dos ítems y el cuarto factor quedó integrado por 5 ítems $(6=a l-$ gunas de las cosas que he vivido me han ayudado a distinguir lo que es importante de lo que no lo es; $8=$ las emociones son una de las cosas que hacen que valga la pena vivir; 19= conozco los motivos por los que cambian mis emociones; $21=$ tengo control sobre mis emociones y $26=$ cuando alguien me cuenta las cosas importantes que ha vivido, siento como si las hubiera vivido yo mismo), que presentaron valores propios (eigenvalues) muy inferiores a .40. Se trató de los mismos ítems que Gignac y sus colegas (2005) encontraron como inclasificables sobre cualquiera de las dimensiones de IE propuestas por Salovey y Mayer (1990). El segundo análisis, efectuado ahora sobre 28 ítems, mostró una solución de dos factores a partir del examen del gráfico de sedimentación. Después de la rotación oblicua, se retuvo la solución bifactorial por ser la de mejor ajuste y significativamente interpretable. En esta oportunidad, se volvieron a descartar los ítems con pesos factoriales menores a .40 y que presentaron un peso factorial mayor a .30 en un segundo factor. Por lo que al final de este proceso se eliminaron 3 ítems, quedando la escala final conformada por 25 reactivos, distribuidos sobre dos factores (tabla 2 ).

Los dos componentes extraídos explicaron el 63.9 $\%$ de la varianza total, no reproduciéndose la solución unidimensional encontrada por Schutte y colegas (1998). El primer componente quedó integrado por 15 ítems referidos a dos de las tres dimensiones conceptuales de Salovey y Mayer (Expresión de las emociones y Regulación emocional), por lo que fue rotulado como Expresión y regulación de las emociones. El segundo componente nucleó 10 ítems, todos vinculados con la dimensión Usos de la emoción, por lo que fue identificado con ese nombre. Con el propósito de confirmar la estructura identificada, se calculó un conjunto de AFC con el auxilio del programa AMOS 15.0. Las medidas de bondad de ajuste calculadas indicaron que los datos se ajustaban razonablemente bien tanto a un modelo de tres factores $\left(\chi^{2}=9345.72, R M S E A=.05, G F I=.92 ; C F I=.93 ; T L I=\right.$ .83) como a uno de dos factores $\left(\chi^{2}=10216.04\right.$, RMSEA $=.04, \mathrm{GFI}=.95 ; \mathrm{CFI}=.94 ; T L I=.91)$. Sin embargo, una comparación de los dos modelos usando la prueba de diferencia de chi-cuadrado $\left(\chi^{2}=1215.70, p<.001\right)$ indicó que el modelo de dos factores se ajustaba mejor a los datos que el modelo de tres factores, apoyando la distinción entre Expresión y regulación de las emociones, y Usos de la emoción. La validez convergente fue explorada mediante el cómputo de coeficientes de correlación (tabla 3) entre los puntajes de los dos factores extraídos (y el puntaje global de IE) y el conjunto de variables descritas en el apartado Instrumentos (Método). 
Tabla 2.

Análisis de componentes principales de los ítems de la Escala de Inteligencia Emocional de Schutte (apaisados en portugués).

\begin{tabular}{|c|c|c|}
\hline & Factor 1 & Factor 2 \\
\hline \multicolumn{3}{|l|}{ Factor 1: Expresión y regulación de las emociones } \\
\hline $\begin{array}{l}\text { 1. Sé por qué cambian mis emociones } \\
\text { Sei por que minhas emoções mudam }\end{array}$ & .88 & \\
\hline $\begin{array}{l}\text { 2. Me doy cuenta cuando experimento una emoción positiva } \\
\text { Consigo perceber quando experimento uma emoção positiva }\end{array}$ & .82 & \\
\hline $\begin{array}{l}\text { 3. Reconozco las emociones de la gente por sus expresiones faciales } \\
\text { Reconheço as emoções das pessoas por suas expressões faciais }\end{array}$ & .76 & \\
\hline $\begin{array}{l}\text { 4. Soy consciente de las emociones que experimento } \\
\text { Sou consciente das emoções que experimento }\end{array}$ & .72 & \\
\hline $\begin{array}{l}\text { 5. Reconozco cómo se sienten los demás a partir de su tono de voz } \\
\text { Reconheço como as pessoas se sentem a partir de seu tom de voz }\end{array}$ & .70 & \\
\hline $\begin{array}{l}\text { 6. Me cuesta interpretar los mensajes no verbales de los demás } \\
\text { Tenho dificuldade para interpretar as mensagens não verbais das pessoas }\end{array}$ & .69 & \\
\hline $\begin{array}{l}\text { 7. Espero hacer bien la mayoría de las cosas que emprendo } \\
\text { Espero fazer bem a maioria das coisas que empreendo }\end{array}$ & .69 & \\
\hline $\begin{array}{l}\text { 8. A la gente le resulta fácil confiar en mí } \\
\text { É fácil para as pessoas confiarem em mim }\end{array}$ & .64 & \\
\hline $\begin{array}{l}\text { 9. Anticipo buenos resultados frente a las cosas que emprendo } \\
\text { Antecipo bons resultados frente as coisas que empreendo }\end{array}$ & .61 & \\
\hline $\begin{array}{l}\text { 10. Cuando cambio el ánimo, veo nuevas posibilidades } \\
\text { Quando mudo o ânimo, vejo novas possibilidades }\end{array}$ & .57 & \\
\hline $\begin{array}{l}\text { 11. Cuando me enfrento a un desafío, pienso que fracasaré } \\
\text { Quando enfrento um desafio, prenso que fracassarei }\end{array}$ & .57 & \\
\hline $\begin{array}{l}\text { 12. Por lo general espero que ocurran cosas buenas } \\
\text { De maneira geral espero que ocorram coisas boas }\end{array}$ & .55 & \\
\hline $\begin{array}{l}\text { 13. Sé en qué momento hablar de mis problemas personales con los demás } \\
\text { Sei em que momento falar de meus problemas pessoais com os demais }\end{array}$ & .54 & \\
\hline $\begin{array}{l}\text { 14. Me doy cuenta de lo que está sintiendo una persona con solo mirarla } \\
\text { Me dou conta do que uma pessoa está sentindo só de olhá-la }\end{array}$ & .53 & \\
\hline $\begin{array}{l}\text { 15. Felicito a los que hacen algo bien } \\
\text { Felicito aos que fazem algo bem }\end{array}$ & .52 & \\
\hline
\end{tabular}

Factor 2: Usos de la emoción

16. Me gusta compartir mis emociones con los demás

Me agrada compartilhar minhas emoções com as pessoas

17. Busco actividades que me hagan feliz Busco atividades que me façam feliz.

18. Cuando estoy de buen humor resuelvo mejor los problemas Quando estou de bom humor rsolvo melhor os problemas

19. Cuando estoy de buen humor soy capaz de tener nuevas ideas Quando estou de bom humor sou capaz de ter novas idéias

20. Cuando me enfrento a un problema, pienso cómo lo resolví antes y actúo de la misma manera Quando enfrento um problema, penso como o resolvi antes e ajo da mesma maneira

21. La gente disfruta de los eventos que organizo As pessoas desfrutam dos eventos que organizo

22. Soy capaz de levantarle el ánimo a los demás Sou capaz de levantar o ânimo das pessoas Trato de causar uma boa impressão aos demais Trato de superar os obstáculos usando meu sentido de humor

25. Cuando cambio de ánimo veo nuevas posibilidades Quando mudo de ânimo vejo novas possibilidades

\begin{tabular}{lrr}
\hline Varianza explicada & 36.20 & 27.70 \\
\hline Coeficiente a de Cronbach & .74 & .86 \\
\hline
\end{tabular}


Tabla 3.

Coeficientes de correlaciones entre las subescalas (y el puntaje total) de la Escala de IE y el resto de las variables exploradas.

\begin{tabular}{|c|c|c|c|c|}
\hline Variables & Subescalas & $\begin{array}{c}\text { Factor } 1 \\
\text { Evaluación y } \\
\text { regulación de } \\
\text { emociones }\end{array}$ & $\begin{array}{l}\text { Factor } 2 \\
\text { Usos de la } \\
\text { emoción }\end{array}$ & $\begin{array}{l}\text { Puntaje Total } \\
\text { Escala IE }\end{array}$ \\
\hline \multirow{3}{*}{ Bienestar Subjetivo } & Bienestar con expectativas y logros & $.219^{* *}$ & $.498^{* *}$ & $.395^{* *}$ \\
\hline & Confianza para afrontar dificultades & $.160^{* *}$ & $.486^{* *}$ & $.348^{* *}$ \\
\hline & Relaciones con el grupo primario & $.109^{* *}$ & $.058^{*}$ & $.078^{*}$ \\
\hline \multirow{3}{*}{ Resiliencia } & Realización personal & $.143^{* *}$ & $.432^{* *}$ & $.251^{* *}$ \\
\hline & Autodeterminación & $.195^{* *}$ & $.224^{* *}$ & $.209^{* *}$ \\
\hline & Autoconfianza & $.172^{* *}$ & $.404^{* *}$ & $.279^{* *}$ \\
\hline \multirow{2}{*}{$\begin{array}{l}\text { Afrontamiento del } \\
\text { estrés }\end{array}$} & Enfocado en el problema & $.323^{* *}$ & $.582^{* *}$ & $.393^{* *}$ \\
\hline & Adaptativo & $.267^{* *}$ & -.041 & $.165^{* *}$ \\
\hline \multirow{2}{*}{ Sentido del humor } & Uso social humor & $.150^{* *}$ & $.313^{* *}$ & $.254^{* *}$ \\
\hline & Uso como coping & $.095^{* *}$ & $-.152^{* *}$ & $.110^{* *}$ \\
\hline
\end{tabular}

${ }^{* *} p<.001 ;{ }^{*} p<.05$

Las tres dimensiones del Bienestar Subjetivo (Bienestar vinculado con expectativas y logros, Confianza para afrontar las dificultades y Relaciones con el grupo primario), presentaron vinculaciones significativas con la IE, tanto con el puntaje global provisto por la escala, como con cada uno de sus dos componentes. Sin embargo, las mayores asociaciones se registraron entre el factor Usos de la emoción y las facetas del bienestar vinculadas expectativas y logros $(r=.498 ; p<.000)$ y confianza para afrontar dificultades $(r=.486 ; p<.000)$.

Por su parte, las tres dimensiones de Resiliencia (Autodeterminación, Realización Personal, y Autoconfianza) también correlacionaron significativa y positivamente tanto con el puntaje global de $\mathrm{IE}$, como con los dos componentes de la escala. En este caso, los coeficientes más elevados se registraron entre el factor Usos de la emoción con Realización Personal $(r=.432 ; p<.000)$ y con Autoconfianza $(r=.404 ; p<.000)$, respectivamente.

En lo que hace a las Estrategias de Afrontamiento del Estrés, tanto las enfocadas en la solución de los problemas, como las adaptativas, se asociaron significativamente con el puntaje total de IE. Las estrategias de afrontamiento activo del problema se vincularon con mayor intensidad con el Uso de las emociones; en tanto que las estrategias adaptativas (tales como buscar apoyo social, rezar y similares), presentaron las mayores correlaciones con la dimensión Evaluación y regulación de las emociones $(r=.267 ; p<.000)$.

En cuanto al sentido del humor, los datos indicaron que el empleo social del humor se vinculó significativa y positivamente con la dimensión Uso de las emociones, mientras que el empleo del humor como estrategia de coping correlaciono negativamente con esta dimensión ( $r=-.152 ; p<.000)$. Se observó, asimismo, correlaciones positivas entre el uso del humor como mecanismo de afrontamiento del estrés y la dimensión Evaluación y regulación de las emociones $(r=.095 ; p<.000)$.

Finalmente, la confiabilidad fue evaluada a través del cálculo de coeficientes de consistencia interna Alpha de Cronbach. Tal como se observa en la Tabla 2, se obtuvieron adecuados valores umbrales para las dos dimensiones. Paralelamente, se calculó la estabilidad del instrumento a lo largo del tiempo (confiabilidad test-retest), administrando 
nuevamente la escala tres semanas después a una muestra de 224 estudiantes (63 argentinos, 80 brasileros y 81 mexicanos). Los coeficientes obtenidos en esta oportunidad fueron de .78 para la escala completa y de .71 y .82 para cada una de sus dos dimensiones, respectivamente.

\section{Discusión}

El propósito del presente estudio fue adaptar y validar la SSREI (Schutte et al. 1998) para su empleo con adolescentes y jóvenes argentinos, mexicanos y brasileros. El análisis de las propiedades psicométricas mostró que la versión adaptada de la escala de IE posee adecuada validez y confiabilidad, lo que la transforma en un instrumento idóneo para su empleo con muestras de la población objetivo.

La revisión bibliográfica muestra que la estructura factorial de la SSREI ha generado resultados controversiales, que van desde soluciones unifactoriales (Brackett y Mayer, 2003; Schutte et al. 1998), a multidimensionales (Ciarrochi et al. 2000; Gignac et al. 2005; Petrides y Furham, 2000). Por tal motivo, muchos autores han subrayado la necesidad de seguir analizando su estructura empleando mayores tamaños muestrales, cosa que se ha hecho en el presente estudio, desde el momento que se ha trabajado con una muestra de casi 2.000 sujetos. En este caso concreto, a través de análisis de componentes principales, con rotación oblicua (tal como lo recomendara Gignac et al. 2005) se identificaron dos posibles modelos de IE. La primera solución, definida por un modelo tetrafactorial, fue descartada porque los ítems que conformaban el tercer y el cuarto factor presentaron pesos factoriales despreciables. En cambio, fue retenida la segunda solución ya que los ítems presentaron muy buen ajuste sobre los dos factores identificados. El primero de ellos nucleó 15 ítems relacionados con dos de las tres facetas definidas en el modelo de IE propuesto por Salovey y Mayer (1990), sobre la que está basada la SSREI. Por lo que este factor fue rotulado como Expresión y regulación de las emociones. El segundo factor agrupó diez ítems referidos a la faceta Usos de la emoción, por lo que se la rotuló con ese mismo nombre. Ambos factores explicaron un porcentaje importante de la varianza del constructo IE (63.9 $\%)$ y mostraron poseer adecuada consistencia interna (coeficientes alpha superiores a .70), y confiabilidad test-retest.

El análisis de la validez convergente mostró asociaciones significativas entre IE y el resto de los constructos medidos (resiliencia, bienestar subjetivo, sentido del humor y estrategias positivas de coping). Tales resultados coinciden tanto con las especulaciones teóricas preliminares como con la evidencia empírica más reciente (Bhullar et al. 2013). Por ejemplo, mientras que la teoría ha sugerido que la IE está vinculada con altos niveles de bienestar subjetivo, y que los sujetos con elevada IE son capaces de mantener estados mentales positivos más largos y más frecuentes (Mayer y Salovey, 1997; Mikolajczak y Luminet, 2008), los estudios empíricos han corroborado consistentemente esta relación positiva entre IE y bienestar. En este sentido, Bar-On (2005) informó correlaciones positivas muy elevadas $(r=.76)$ entre los dos constructos, mientras que Higgs y Dulewicz (2014) encontraron que la IE era el mejor predictor de la varianza total del bienestar general. Estas relaciones también han sido encontradas por Carmeli, Yitzhak-Halevy y Wisberg (2009) en su estudio orientado a examinar las relaciones entre IE y algunas dimensiones del bienestar (autoaceptación, satisfacción y autoestima), al informar coeficientes de correlación que variaron entre .22 y .46 . Este conjunto de evidencias ha llevado a Kämpfe y Mitte (2010) a señalar que las características de la IE que más contribuyen al bienestar son las siguientes: (a) la capacidad de comprender y aceptar las propias emociones y las de los demás, (b) la capacidad de establecer y alcanzar metas personales para mejorar el propio potencial, y (c) la capacidad de verificar los propios sentimientos y poner las cosas en la perspectiva correcta. Esto significa que las personas con un mayor sentido de bienestar son las que poseen autoconocimiento emocional, autoestima positiva, autorealización, y evaluación efectiva de la realidad. Tales señalamientos coinciden con los efectuados con anterioridad por otros investigadores utilizando diferentes medidas de IE (Brackett y Mayer, 2003). En síntesis, los hallazgos emergentes de esta línea 
de estudios han permitido concluir que los sujetos con alta IE tienen habilidades sociales y estrategias de sobrevivencia más efectivas, lo que favorece un aumento de su sentido de bienestar.

A su vez, existe un creciente cuerpo de evidencia empírica que sugiere que la IE impacta significativamente sobre otras formas de comportamiento tales como la capacidad de hacer frente a los problemas y ser resiliente frente a las dificultades cotidianas (Bar-On, 2005). Las significativas relaciones encontradas en el presente trabajo entre IE y resiliencia, están en sintonía con las obtenidas por Omar (2013), quien señala que los jóvenes emocionalmente más inteligentes tienen la capacidad de transformar las emociones negativas en crecimiento positivo frente a las adversidades. Coinciden también con los hallazgos comunicados por Liu et al. (2013), cuando subrayan que la capacidad de evaluar, regular y usar adecuadamente las emociones favorece la resiliencia. Sintetizando los estudios que han analizado las relaciones entre IE y resiliencia, se puede señalar que las mayores contribuciones de la IE a la resiliencia son las siguientes: (a) la capacidad de conocerse y aceptarse; (b) la capacidad de ser consciente de los sentimientos de los demás, (c) la capacidad de manejar las emociones, y (d) la posibilidad de tener una actitud positiva y optimista.

Otras evidencias de la validez convergente de la SSREI surgen a la luz de sus correlaciones con las estrategias de afrontamiento del estrés. En este sentido, si bien los dos factores de la IE se asociaron positivamente con las estrategias de afrontamiento activo del problema, el factor Usos de la emoción no mostró relaciones significativas con el empleo de estrategias adaptativas. Estos resultados coinciden con los estudios de Wu (2011), quien señala que el afrontamiento focalizado en el problema es patrimonio de las personas que usan eficazmente su IE, así como con los de Davis y Humphrey (2012), quienes han indicado que las estrategias adaptativas se asocian más con bienestar que con IE. Finalmente, con respecto a las relaciones entre IE y sentido del humor, se observaron asociaciones positivas entre la dimensión Evaluación y regulación de las emociones, tanto con el uso social del humor como con el empleo del hu- mor como estrategia de coping, coincidiendo con lo adelantado previamente por Karou-ei, Doosti, Dehshiri y Heidari (2009). Sin embargo, la faceta Usos de la emoción presentó asociaciones negativas con el empleo del humor como estrategia de afrontamiento del estrés. Hallazgo en línea con lo comunicado por Omar (2013), cuando señala que por tratarse de jóvenes y adolescentes, todavía no han aprendido a usar el humor para afrontar dificultades, superar adversidades o, simplemente, para distender situaciones embarazosas.

En cuanto a las implicancias prácticas que se pueden derivar del presente trabajo, además de seguir explorando cómo se desarrolla la IE a lo largo de la vida, sería importante examinar el impacto de la IE sobre la crianza y educación de niños y adolescentes bien adaptados, eficaces y productivos. Ya que así como la IE se puede mejorar a través de métodos didácticos simples en un periodo relativamente corto de tiempo (Bar-On, 2007), también sería valioso examinar la mejor manera de aumentar las competencias y habilidades emocionales desde temprana edad. Al respecto, sería deseable desarrollar programas educativos orientados a mejorar los comportamientos inteligentes emocional y socialmente basados en evidencias empíricas, más que en suposiciones y elucubraciones sin fundamento. El desarrollo de estos programas se debería basar en los resultados de las investigaciones que muestran los efectos de la IE sobre los más variados aspectos de rendimiento humano, la autorrealización, la felicidad y el bienestar. Es razonable suponer que si se tiene éxito en la crianza y educación con mayor énfasis en los aspectos sociales y emocionales, se estará contribuyendo a la construcción de organizaciones, comunidades y sociedades más eficaces, productivas y humanas, que es uno de los principales objetivos del movimiento de la psicología positiva.

\section{Conclusión}

A modo de colofón se puede adelantar que las propiedades psicométricas de la versión adaptada de la SSREl (Schutte et al. 1998), lo transforman en un instrumento apto para su empleo con jóvenes y adolescentes latinoamericanos. La versión 
adaptada mostró una adecuada consistencia interna, una aceptable estabilidad a través del tiempo, y que el lenguaje con el que están redactados los ítems demanda una comprensión lectora acorde con la edad de la población objetivo. El análisis de componentes principales indicó una solución de dos factores, que explicaron cerca del $64 \%$ de la varianza de la IE; estructura que fue confirmada mediante el cálculo de un análisis factorial confirmatorio. Las significativas correlaciones entre los factores de la IE identificados y medidas de bienestar subjetivo, resiliencia, sentido del humor y estrategias de afrontamiento del estrés, dieron apoyo a la validez convergente de la escala.

\section{Agradecimientos}

Los autores agradecen a la doctora Pino Peña y a los doctores Aguiar de Souza y Almeida da Silva Junior, por su colaboración en la recolección de datos correspondientes a las muestras mexicanas y brasileras. Parte de este trabajo fue realizado con el apoyo financiero (PIP 112-201101000001) otorgado a la primera autora por el CONICET (Argentina).

\section{Referencias}

Almeida, S. H., Aguiar, M., Monteiro, N., y Omar, A. (24 de octubre, 2007). Avaliação da resiliência, valores e do humor em estudantes: um estudo Brasil-Argentina. Trabajo presentado en la XXXVII Reunión Anual de la Sociedad Brasilera de Psicología. Brasil: Florianópolis, Santa Catarina.

American Psychological Association. (1999). Standards for educational and psychological testing. Washington, D.C.: Author.

Austin, E. J., Saklofske, D. H., Huang, S. H., y McKenney, D. (2004). Measurement of trait emotional intelligence: Testing and cross validating a modified version of Schutte et al.'s (1998) measure. Personality and Individual Differences, 36(3), 555-562. DOI: 10.1016/ S0191-8869(03)00114-4.
Bar-On, R. (1997). The Emotional Quotient Inventory (EQ-i): A test of emotional intelligence. Toronto, Canadá: Multi-Health Systems, Inc.

Bar-On, R. (2005). The Bar-On model of emotionalsocial intelligence (ESI). Issues in Emotional Intelligence, 1, 1-28.

Bar-On, R. (2007). How important is it to educate people to be emotionally intelligent and can it be done? In R. Bar-On, J.G. Maree y M. Elias (eds.). Educating people to be emotionally intelligent, 1-14. Santa Barbara, California: Greenwood Pub Group.

Bhullar, N., Schutte, N. S., y Malouff, J. M. (2013). The nature of well-being: The roles of hedonic and eudaimonic processes and trait emotional intelligence. The Journal of Psychology: Interdisciplinary and Applied, 147, 1-16. DOI: 10.1080/00223980.2012.667016.

Brackett, M. A., y Mayer, J. D. (2003). Convergent, discriminant, and increment validity of competing measures of emotional intelligence. Personality and Social Psychology Bulletin, 29, 1147-1158. DOI: 10.1177/0146167203254596.

Carmeli, A., Yitzhak-Halevy, M., y Wisberg, (2009). The relationship between emotional intelligence and psychological wellbeing. Journal of Managerial Psychology, 24(1), 66-78. DOI: 10.1108/02683940910922546.

Chan, D. W. (2003). Dimensions of emotional intelligence and their relationships with social coping among gifted adolescents in Hong Kong. Journal of Youth \& Adolescence, 32(6), 409418. DOI: 10.1023/A:1025982217398.

Ciarrochi, J., Chan, A., y Caputi, P. (2000). A critical evaluation of the emotional intelligence construct. Personality and Individual Differences, 28, 539-561. DOI: 10.1016/S01918869(00)00108-2.

Davis, S. K., y Humphrey, N. (2012). Emotional intelligence as a moderator of stressor-mental health relations in adolescence: Evidence for specificity. Personality and Indivi- 
dual Differences, 52, 100-105. DOI: 0.1016/j. paid.2011.09.006.

Fiori, M., y Antonakis, J. (2011). The ability model of emotional intelligence: Searching for valid measures. Personality and Individual Differences, 50(3), 329-334. DOI: 10.1016/j. paid.2010.10.010.

Frydenberg, E., y Lewis, R. (1997). Manual de Escalas de afrontamiento para adolescentes. Madrid, España: TEA.

García-Coll, V., Graupera-Sanz, J. L., Ruiz-Pérez, L. M., y Palomo-Nieto, M. (2013). Inteligencia emocional en el deporte: Validación española del Schutte self Report Inventory (SSRI) en deportistas españoles. Cuadernos de Psicología del Deporte, 13(1), 25-36. DOI: 10.4321/ S1578-84232013000100004.

Gignac, G. E., Palmer, B. R., Manocha, R., y Stough, C. (2005). An examination of the factor structure of the Schutte Self-report Emotional Intelligence (SSREI) scale via confirmatory factor analysis. Personality and Individual Differences, 39, 1029-1042. DOI: 10.1016/j.paid.2005.03.014.

Goleman, D. (1995). Emotional Intelligence. New York: Bantam Books.

Hair, J. F., Black, B., Babin, B., y Anderson, R. E. (2009). Multivariate data analysis $\left(7^{\text {th }}\right.$ ed.).Upper Saddle River, NJ: Pearson Prentice Hall.

Hakanen, E. A. (2004). Relation of emotional intelligence to emotional recognition and mood management. Psychological Reports, 94(3), 97-103.

Herdman, M., Fox-Rushby, J., y Badia, X. (1998). A model of equivalence in the cultural adaptation of HRQoL instruments: The universalist approach. Quality of life Research, 7(4), 323335. DOI: 10.1023/A:1008846618880.

Higgs, M., y Dulewicz, V. (2014). Antecedents of well-being: A study to examine the extent to which personality and emotional intelligence contribute to well-being. International Journal of Human Resource Management, 25(5), 718-735. DOI: 10.1080/09585192.2013.815253.

Kämpfe, N., y Mitte, K. (2010). Tell me who you are, and I will tell you how you feel? European Journal of Personality, 24, 291-308. DOI: 10.1002/per.743.

Karou-ei, R., Doosti, Y., Dehshiri, G., y Heidari, M. (2009). Humor styles, subjective well-being, and emotional intelligence in college students. Journal of Iranian Psychologists, 5(18), 159-169.

Liu, Y., Wang, Z., y Lü, W. (2013). Resilience and affect balance as mediators between trait emotional intelligence and life satisfaction. Personality and Individual Differences, 54(7), 850-855. DOI: 10.1016/j.paid.2012.12.010.

Mayer, J. D., y Salovey, P. (1993). The intelligence of emotional intelligence. Intelligence, 17(4), 433-442. DOI: 10.1016/0160-2896(93)90010-3.

Mayer, J. D., y Salovey, P. (1997). What is emotional intelligence? En P. Salovey y D. Sluyter (eds.). Emotional development and emotional intelligence: Educational implications, 3-31. New York: Basic Book.

Mayer, J. D., Caruso, D. R., y Salovey, P. (1999). Emotional intelligence meets traditional standards for an intelligence. Intelligence, 27, 267-298. DOI: 10.1016/S0160-2896(99)00016.

Mayer, J. D., Roberts, R. D., y Barsade, S. G. (2008). Human abilities: Emotional intelligence. Annual Review of Psychology, 59, 507-536. DOI: 10.1146/annurev. psych.59.103006.093646.

Mayer, J. D., Salovey, P., y Caruso, D. R. (2002). Mayer-Salovey-Caruso Emotional Intelligence Test (MSCEIT) user's manual. Toronto: MHS Publishers.

Mayer, J. D., Salovey, P., y Caruso, D. R. (2012). The validity of the MSCEIT: Additional analy- 
ses and evidence. Emotion Review, 4(4), 403408. DOI: 10.1177/1754073912445815.

Mikolajczak, M., y Luminet, O. (2008). Trait emotional intelligence and the cognitive appraisal of stressful events: An exploratory study. Personality and Individual Differences, 44, 14451453. DOI: $10.1177 / 0143034309360436$.

Nacpal, A., y Shell, B. (1992). Global Well-being Inventory. Nueva Delhi, India: OMS.

Omar, A. (2013). Predictores de resiliencia en jóvenes y adolescentes. En J. Gaxiola, y J. Palomar (eds.), Estudios de resiliencia en América Latina, 2, 19-35. México: Pearson Educación.

Omar, A., Paris, L., Aguiar, M., Almeida, S. H., y Pino Peña, R. (2009). Validación del Inventario de Bienestar Subjetivo con muestras de jóvenes y adolescentes argentinos, brasileros y mexicanos. Suma Psicológica, 16(2), 69-84.

Petrides, K. V., y Furnham, A. (2000). On the dimensional structure of emotional intelligence. Personality and Individual Differences, 29, 313-320. DOI: 10.1016/S0191-8869(99)00195-6.

Petrides, K. V., y Furnham, A. (2003). Trait emotional intelligence: Behavioral validation in two studies of emotion recognition and reactivity to mood induction. European Journal of Personality, 17(1), 39-57. DOI: 10.1002/ per.466.

Roberts, R. D., MacCann, C., Matthews, G., y Zeidner, M. (2010). Emotional intelligence: Toward a consensus of models and measures. Social and Personality Psychology Compass, 4(10), 821-840. DOI: 10.1111/j.17519004.2010.00277.x.

Saklofske, D. H., Austin, E. J., Mastoras, S. M., Beaton, L., y Osborne, S. E. (2012). Relationships of personality, affect, emotional intelligence and coping with student stress and academic success: Different patterns of association for stress and success. Learning and Individual Differences, 22, 251-257. DOI: 10.1016/j.lindif.2011.02.010.

Salovey, P., y Mayer, J. D. (1990). Emotional intelligence. Imagination, Cognition and Personality, 9, 185-211. DOI: 0.2190/DUGG-P24E-52WK6CDG.

Schutte, N. S., Malouff, J. M., Hall, L. E., Haggerty, D. J., Cooper, J. T., Golden, C. J. et al. (1998). Development and validation of a measure of emotional intelligence. Personality and individual differences, 25(2), 167-177. DOI: 10.1016/S0191-8869(98)00001-4.

Thorson, J. A., y Powell, F. C. (1993). Development and validation of a multidimensional sense of humor scale. Journal of Clinical Psychology, 49(1), 13-23. DOI: 10.1002/1097-4679(199301)49:1<13::AIDJCLP2270490103>3.0.CO;2-S

Van Rooy, D. L., Whitman, D. S., y Viswesvaran, C. (2010). Emotional intelligence: Additional questions still unanswered. Industrial and Organizational Psychology: Perspectives on Science and Practice, 3(2), 149-153. DOI: 10.1111/j.1754-9434.2010.01216.x.

Wagnild, G. M., y Young, H. M. (1993). Development and psychometric evaluation of the Resilience Scale. Journal of Nursing Measurement, 1(2), 165-178.

Wu, Y. C. (2011). Job stress and job performance among employees in the Taiwanese finance sector: The role of emotional intelligence. Social Behavior and Personality: An International Journal, 39(1), 21-31. DOI: 10.2224/ sbp.2011.39.1.21. 